\title{
Significant protection from infection and AIDS progression after gastrointestinal and oral vaccinations, respectively, with a SIV DNA/rMVA vaccine
}

\author{
A Aldovini ${ }^{1 *}$, M Manrique ${ }^{1}$, A Cobo-Molinos ${ }^{1}$, P Kozlowski ${ }^{2}$, A Carvlle $^{3}$ \\ From AIDS Vaccine 2012 \\ Boston, MA, USA. 9-12 September 2012
}

\section{Background}

Nasal SIV vaccination can significantly protect from AIDS progression.

\section{Methods}

We compared four mucosal routes of vaccination in four groups of seven female Rhesus Macaques (RM) each, immunized in the oral cavity $(\mathrm{O})$, gastrointestinally $(\mathrm{GI})$, nasally $(\mathrm{N})$ and vaginally $(\mathrm{V})$ with mutated proviral SIV, IL-2 and IL-15 DNAs and SIV rMVA. Vaccinated and control animals were challenged vaginally with repeated low-dose of SIVmac251.

\section{Results}

Only $\mathrm{N}$ vaccination induced a significant increase in plasma SIV-IgG titers. Significantly higher systemic, rectal and vaginal SIV-specific $\mathrm{T}$-cell responses were detected in the oral group during the immunization. The median number of challenges required to become infected was significantly higher in the GI group (32; 16 for $\mathrm{O}, 12$ for $\mathrm{V}$, 9 for N, 11 for controls). Repeated SIV exposure expanded vaginal anti-SIV T-cells in some of the animals. Seven vaccinated RM (3 in the $\mathrm{O}, 3$ in the $\mathrm{N}$ and 1 in the GI group) suppressed the viremia after the initial infection peak and maintained it undetectable over the course of the trial. Immunized, infected animals had significantly lower levels of systemic $\mathrm{T}$-cell immune activation, better preservation of CD $4+$ central memory and $\alpha 4 \beta 7$ high $+C D 4+\mathrm{T}$-cells, with consequent better protection from AIDS. However a lower protection from AIDS progression was observed in the GI group compared to the other vaccinated RM, with a median survival of 24 weeks. A significantly higher loss of CD4+ CM T-cells, detected early on in this group, correctly predicted its poor long-term outcome.

\section{Conclusion}

Protection from infection in The GI group correlated with higher anti-SIV CD8+ T cells responses in vaginal $\mathrm{T}$-cells on the day of first challenge. More than $50 \%$ of the $\mathrm{O}$ and $\mathrm{N}$ vaccinated $\mathrm{RM}$ were still disease-free 72 weeks after infection, and protection correlated with levels of systemic anti-SIV IFN-g+/CD8+ T-cells on the day of first challenge.

\section{Author details}

'Children's Hospital Boston, Harvard Medical School, Boston, MA, USA. ${ }^{2}$ Louisiana State University Health Sciences Center, New Orleans, LA, USA. ${ }^{3}$ New England Primate Research Center, Harvard Medical School, Southborough, MA, USA

Published: 13 September 2012

\section{doi:10.1186/1742-4690-9-S2-O}

Cite this article as: Aldovini et al:: Significant protection from infection and AIDS progression after gastrointestinal and oral vaccinations, respectively, with a SIV DNA/rMVA vaccine. Retrovirology 2012 9(Suppl 2): 01. 